\title{
Drug-coated balloon treatment in coronary artery disease: Recommendations from an Asia-Pacific Consensus Group
}

\author{
Ae-Young Her ${ }^{1}$, Eun-Seok Shin ${ }^{2}$, Liew Houng Bang ${ }^{3}$, Amin Ariff Nuruddin', \\ Qiang Tang ${ }^{5}$, I-Chang $\mathrm{Hsieh}^{6}$, Jung-Cheng $\mathrm{Hsu}^{7}$, Ong Tiong Kiam ${ }^{8}$, \\ ChunGuang Qiu ${ }^{9}$, Jie Qian ${ }^{10}$, Wan Azman Wan Ahmad ${ }^{11}$, Rosli Mohd Ali ${ }^{12}$
}

${ }^{1}$ Division of Cardiology, Department of Internal Medicine, Kangwon National University School of Medicine, Chuncheon, South Korea; ${ }^{2}$ Division of Cardiology, Department of Internal Medicine, Ulsan Medical Center, Ulsan, South Korea; ${ }^{3}$ Cardiology Department and Clinical Research Center, Queen Elizabeth Hospital II,

Kota Kinabalu, Malaysia; ${ }^{4}$ Cardiology Department, National Heart Institute Malaysia, Kuala Lumpur, Malaysia; ${ }^{5}$ Division of Cardiology, Department of Internal Medicine, Beijing University ShouGang Hospital, Beijing, China; ${ }^{6}$ Department of Cardiology, Chang Gung Memorial Hospital, Linkou, Taoyuan, Taiwan; ${ }^{7}$ Department of Cardiology, Far Eastern Memorial Hospital, New Taipei, Taiwan; ${ }^{8}$ Cardiology Department, Sarawak Heart Center, Kota Samarahan, Malaysia; ${ }^{9}$ Division of Cardiology, Department of Internal Medicine, The First Affiliated Hospital of ZhengZhou University, ZhengZhou, China; ${ }^{10}$ Division of Cardiology, Department of Internal Medicine, Beijing FuWai Hospital, Beijing, China; ${ }^{11}$ Division of Cardiology, Department of Medicine, University Malaya Medical Center, Kuala Lumpur, Malaysia;

${ }^{12}$ Cardiac Vascular Sentral Kuala Lumpur, Kuala Lumpur, Malaysia

\begin{abstract}
Coronary artery disease (CAD) is currently the leading cause of death globally, and the prevalence of this disease is growing more rapidly in the Asia-Pacific region than in Western countries. Although the use of metal coronary stents has rapidly increased thanks to the advancement of safety and efficacy of newer generation drug eluting stent (DES), patients are still negatively affected by some the inherent limitations of this type of treatment, such as stent thrombosis or restenosis, including neoatherosclerosis, and the obligatory use of dual antiplatelet therapy (DAPT) with unknown optimal duration.

Drug-coated balloon (DCB) treatment is based on a leave-nothing-behind concept and therefore it is not limited by stent thrombosis and long-term DAPT; it directly delivers an anti-proliferative drug which is coated on a balloon after improving coronary blood flow. At present, DCB treatment is recommended as the first-line treatment option in metal stent-related restenosis linked to DES and bare metal stent. For de novo coronary lesions, the application of DCB treatment is extended further, for conditions such as small vessel disease, bifurcation lesions, and chronic total occlusion lesions, and others. Recently, several reports have suggested that fractional flow reserve guided DCB application was safe for larger coronary artery lesions and showed good long-term outcomes. Therefore, the aim of these recommendations of the consensus group was to provide adequate guidelines for patients with CAD based on objective evidence, and to extend the application of DCB to a wider variety of coronary diseases and guide their most effective and correct use in actual clinical practice. (Cardiol J 2021; 28, 1: 136-149)
\end{abstract}

Key words: drug-coated balloon, Asia-Pacific, coronary artery disease, de novo lesion, in-stent restenosis

Address for correspondence: Eun-Seok Shin, MD, PhD, Division of Cardiology, Department of Internal Medicine, Ulsan Medical Center, 13, Wolpyeong-ro 171beon-gil, Nam-gu, Ulsan, 44686, South Korea, tel: 82-52-250-5020, fax: 82-52-259-5117, e-mail: sesim1989@gmail.com

Received: 3.08.2019 Accepted: 17.09.2019

This article is available in open access under Creative Common Attribution-Non-Commercial-No Derivatives 4.0 International (CC BY-NC-ND 4.0) license, allowing to download articles and share them with others as long as they credit the authors and the publisher, but without permission to change them in any way or use them commercially. 


\section{Introduction}

Non-stent based local drug delivery using a drug-coated balloon (DCB) has emerged as a new treatment modality for coronary artery disease (CAD) [1]. The proposed advantages of DCB include homogeneous drug delivery to the vessel wall, immediate drug release without the use of a polymer, the potential of reducing the intensity and duration of antiplatelet therapy, and the absence of residual foreign material in the vessel [2]. Current DCB treatment has an established indication in the lesion of in-stent restenosis (ISR) and small vessel disease, but there is a need for more data regarding other variable disease subsets. Although several published data from registries and randomized trials provided the empirical basis for the current European and German guidelines $[2,3]$, the demographics and patterns of disease are different in patients of the Asia-Pacific region when compared to those in Europe. Asia-Pacific patients have relatively smaller coronary arteries but longer lesion length as observed by coronary angiography, when compared to Western patients [4]. This coronary phenotype of "small and diffuse CAD" could be a reflection of the higher rate of diabetes mellitus in Asia-Pacific patients. Small vessel CAD carries a worse prognosis than large vessel CAD in terms of restenosis as it is less capable of accommodating neointimal growth after stenting [5]. Another difference is that, in the contemporary trials of antithrombotic treatment, Asia-Pacific patients have a higher risk for bleeding (especially, gastrointestinal bleeding and hemorrhagic stroke) [6-8]. Therefore, the aim of these recommendations of the consensus group is to provide adequate guidelines for Asia-Pacific patients with CAD based on the objective evidence and extend the application of DCB to a wider variety of coronary diseases and guide their most effective and correct use in real clinical practice.

\section{Drug-coated balloon application for coronary artery disease}

\section{In-stent restenosis}

Historically, there have been many questions concerning whether the plain old balloon angioplasty (BA) or stent-in-stent approach is the best option for the treatment of ISR. Previous trials reported that treatment using bare metal stent (BMS)-ISR and drug-eluting stent (DES)-ISR with BA or first-generation DES still resulted in high revascularization rates and long-term stent thrombosis rates compared to DCB [9-12]. Even in the era of newer generation DES with enhanced performance, ISR is still clinically challenging. In this regard, the use of DCB has been proven to be very effective in patients with both BMS-ISR and DES-ISR.

Bare-metal stent restenosis. The Paccocath ISR-I trial of BMS-ISR demonstrated, for the first time, that DCB was superior to BA alone [13]. The angiographic results and the rate of major adverse cardiovascular events (MACE) were significantly improved in the DCB group at follow-up. The larger randomized PEPCAD II trial compared DCB to paclitaxel-DES treatment in BMS-ISR [14]. At 6-month follow-up, DCB significantly reduced the primary endpoint of the study (late lumen loss [LLL]: $0.17 \pm 0.42 \mathrm{~mm}$ in DCB vs. $0.38 \pm 0.61 \mathrm{~mm}$ in DES, $p=0.03)$. These results showed that DCB was at least as efficacious and as well tolerated as first-generation DES in BMS-ISR lesions. The RIBS V trial compared DCB with secondgeneration DES in patients with BMS-ISR [15]. This trial showed better late angiographic findings in the DES group (minimal lumen diameter [MLD]: $2.01 \pm 0.60 \mathrm{~mm}$ in DCB vs. $2.36 \pm 0.60 \mathrm{~mm}$ in DES, $p<0.001$ ), but showed similar rates of restenosis and clinical outcomes. Therefore, the overall non-inferior outcomes of DCB treatment when compared with the outcomes of DES implantation support the use of DCB for the treatment of BMS-ISR lesions.

Drug-eluting stent restenosis. An initial small randomized study demonstrated that DCB provided superior clinical and angiographic results compared with BA alone in patients with DES-ISR at 6-month follow-up [16]. Thereafter, the efficacy of DCB compared to BA in DES-ISR was confirmed in a multicenter, randomized PEPCAD-DES trial including patients with any type (either - limus- or paclitaxel-eluting stents) of DES-ISR [17]. Another controlled PEPCAD China ISR study suggested that DCB is equivalent to paclitaxel-DES in patients with DES-ISR at 9-month follow-up [18]. The larger randomized ISAR-DESIRE 3 trial investigated the efficacy of DCB versus paclitaxel-DES versus BA alone in patients with DES-ISR [19]. The results showed that $\mathrm{DCB}$ was non-inferior to paclitaxel-DES and that both DCB and DES were superior to BA alone at 6 to 8-month follow-up. In summary, the data from the meta-analyses of available randomized clinical trials suggested that DCB is superior to BA alone and is similar to firstgeneration DES in terms of clinical outcomes in patients with DES-ISR [20-22]. 
In the RIBS IV trial, which compared secondgeneration DES to DCB for treatment of DES-ISR, both angiographic and clinical outcomes favored second-generation DES over DCB at 6-9-month follow-up [23]. However, in a recent DARE trial, DCB treatment was comparable to second-generation DES in terms of 6-month MLD (6-month MLD: $1.71 \pm 0.51 \mathrm{~mm}$ in DCB vs. $1.74 \pm 0.61 \mathrm{~mm}$ in DES, $p$ for non-inferiority $<0.0001$ ) and target vessel revascularization (TVR) up to 1 year $(8.8 \%$ in DCB vs. $7.1 \%$ in DES, $p=0.65$ ) in patients with any type of ISR [24]. Therefore, in the future, consideration should be given to ensure randomization when either DCB or DES treatment is possible after BA. In recurrent DES-ISR, DCB and secondgeneration DES yielded similar clinical outcomes (target lesion revascularization [TLR], MACE) at 12-24 months [25]. In a recently published ISAR DESIRE 4 randomized trial, the efficacy of DCB was further improved by optimal lesion preparation by scoring/cutting the balloon [26]. Compared with conventional treatment, scoring balloon predilation was shown to have significantly lowered the primary endpoint rates (diameter stenosis [DS]: $35.0 \pm 16.8 \%$ vs. $40.4 \pm 21.4 \%, \mathrm{p}=0.047$ ) and binary angiographic restenosis rates $(18.5 \%$ vs. $32.0 \%, p=0.026$ ). The latest European Society of Cardiology/European Association for CardioThoracic Surgery (ESC/EACTS) Guidelines on myocardial revascularization recommend the use of DCB for the treatment of both BMS-ISR and DES-ISR lesions that are class I (level of evidence A) [3]. The previous clinical trials are summarized in Table 1.

Therefore, it is recommended that DES-ISR lesions be treated with $\mathrm{DCB}$ if the angiographic results are good after BA, and if otherwise, they should be treated with newer generation DES.

\section{De novo lesion}

Although the combination of DCB with routine BMS implantation resulted in improved outcomes when compared to $\mathrm{BA}$, previous randomized trials using DCB with the routine BMS strategy did not show improvement over a BMS-only approach and was inferior to DES $[27,28]$. The randomized DEB-AMI trial, which enrolled patients treated with DCB plus BMS versus BMS-only versus DES for ST-segment elevation myocardial infarction (STEMI) demonstrated that DCB plus routine BMS was not superior to BMS-only and was inferior to DES [27]. The PEPCAD-III trial also showed that the combination of DCB and BMS failed to prove non-inferiority to sirolimus DES with higher ISR rates $(19 \%$ in $\mathrm{DCB}$ plus BMS vs. $11 \%$ in DES, $\mathrm{p}<0.01$ ) [28]. Therefore, the non-inferiority of routine combination of DCB and BMS in de novo coronary disease is in doubt and recent clinical trials performed a DCB-only approach, reserving stenting for cases in which a suboptimal result was achieved with the DCB-only approach.

Small vessel disease. The DCB-only approach may have an important role in settings such as small vessel disease, because lumen loss after stent implantation comprises a larger percentage of the total lumen diameter in small vessels than in large vessels. Although published evidence for the DCB treatment for small vessel disease is limited, some larger registry data and randomized trial data suggested low MACE rates with DCB use for small vessel disease (diameter of $2 \mathrm{~mm}$ to less than $3 \mathrm{~mm}$ ) (Table 2) [29-33]. In the initial PICCOLETO study comparing DCB with first-generation DES for small vessel disease, DCB was inferior to DES showing a higher percentage of DS than DES at 6-month follow-up [29]. In the PEPCAD I study, 120 patients with small coronary vessels $(2.25-2.8$ $\mathrm{mm}$ ) were treated with the DCB [32]. The patients treated with DCB only had an LLL of $0.18 \pm 0.38$ $\mathrm{mm}$. However, when DCB was combined with bailout BMS, the LLL increased significantly to $0.73 \pm$ $\pm 0.74 \mathrm{~mm}, \mathrm{p}<0.001$. At $12-36$ months follow-up, both the MACE rates and TLR rates increased in the DCB plus BMS group. These results were attributed to the "geographic mismatch phenomenon". In addition, the results suggested the importance of covering the whole dilated segment with the DCB to avoid geographic mismatch. Based on this evidence, the routine use of the combination of DCB and BMS in de novo coronary disease is not recommended. Exceptionally, if the DCB-only approach shows a suboptimal result such as flow-limiting dissection or acute recoil, bail-out BMS implantation should be considered to avoid geographical mismatch. However, recent data has shown that bail-out stenting with DES for suboptimal DCB results is a feasible and safe strategy and is comparable to bail-out BMS $[34,35]$. For this issue, further large-scaled, randomized controlled trials are needed. Recently, a large randomized BASKET-SMALL 2 trial compared DCB with second-generation DES in small CAD using a 12-month composite clinical endpoint of MACE in an all-comer population [33]. A total of 758 patients with de novo lesion ( $<3 \mathrm{~mm}$ in diameter) were randomly enrolled. After 12 months, the rates of MACE were similar in both groups $(7.5 \%$ in DCB vs. $7.3 \%$ in DES; hazard ratio [HR]: 0.97; 95\% confidence interval [CI] 0.58-1.64, $\mathrm{p}=0.918$ ). Rates of 


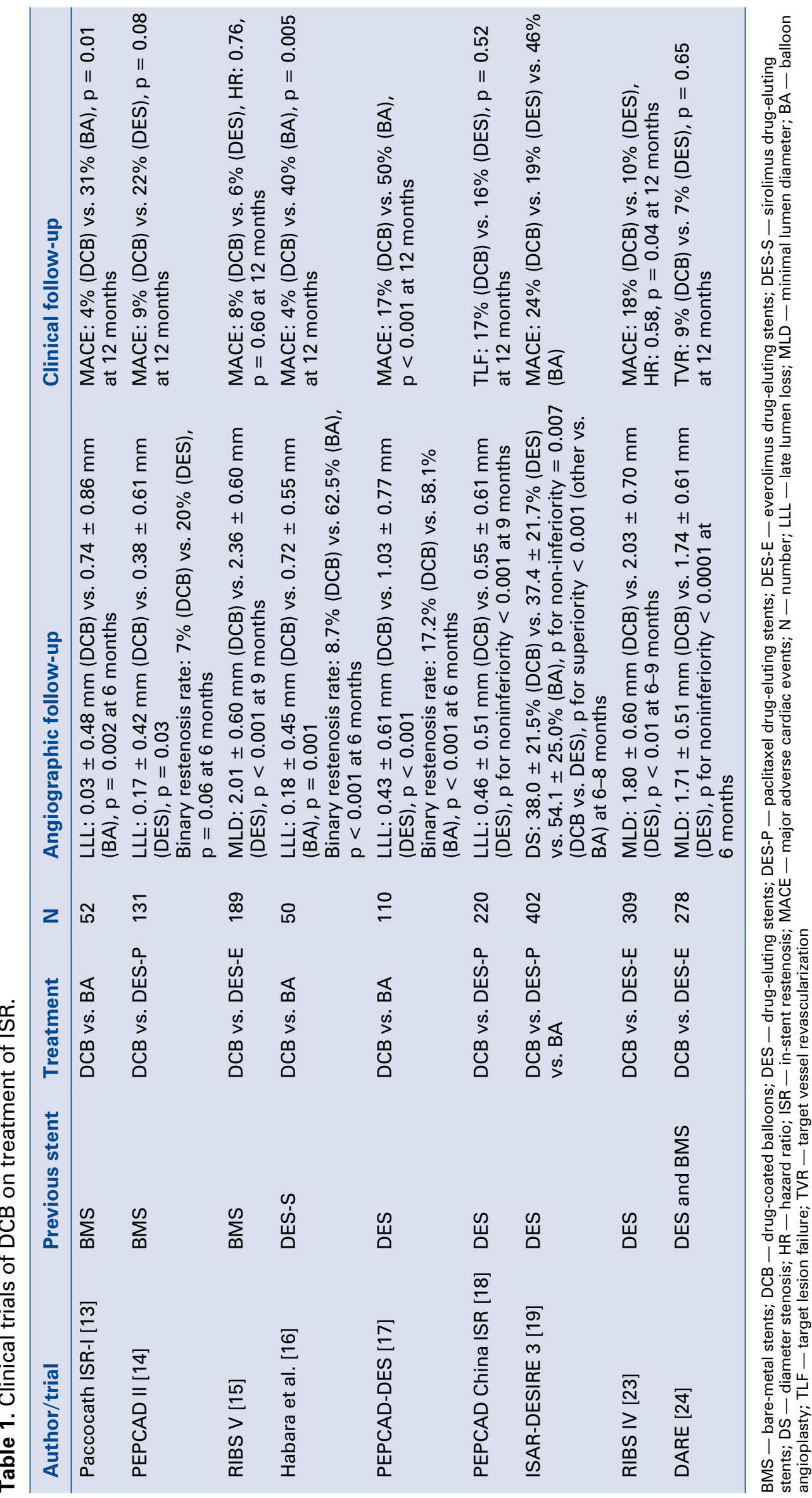




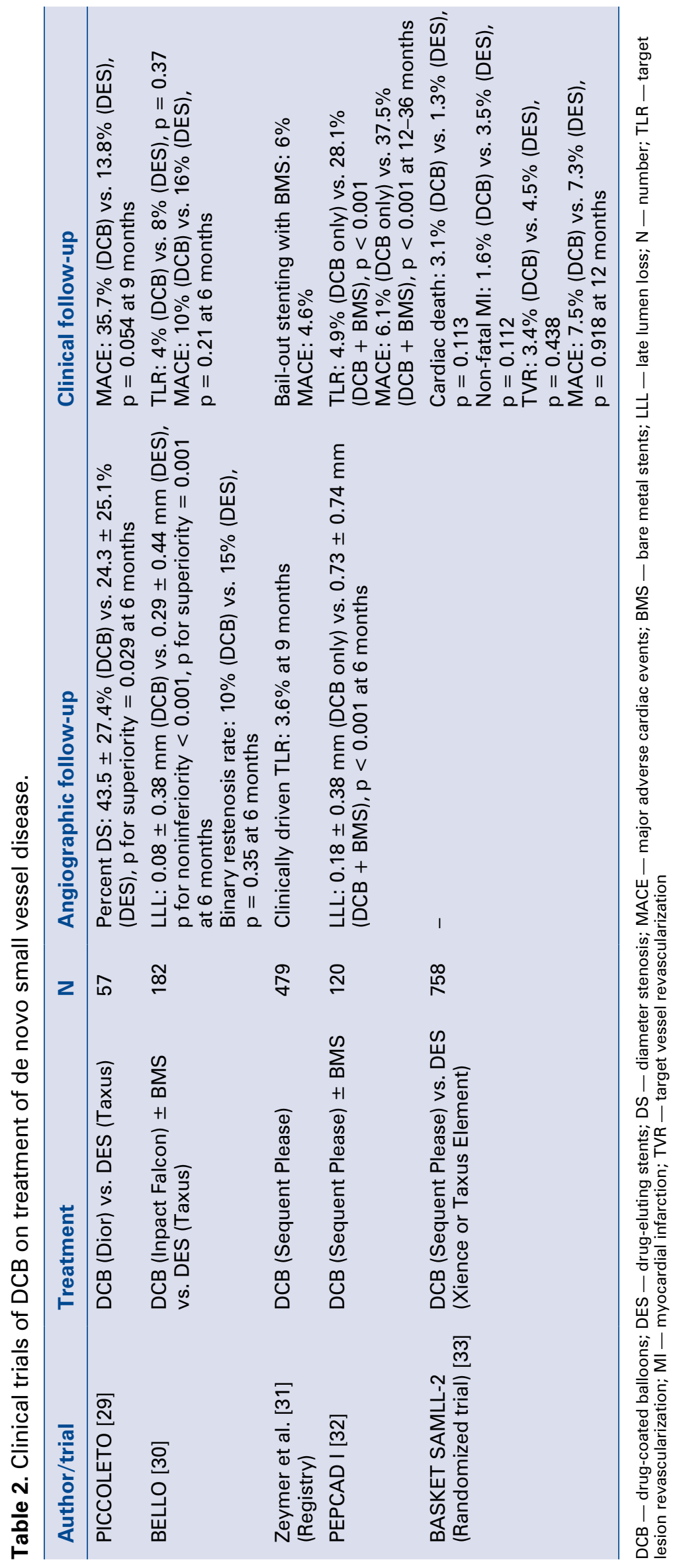


cardiac death, non-fatal myocardial infarction [MI], and TVR did not differ between the groups. The results showed that in small CAD, DCB was noninferior to DES regarding MACE up to 12 months with similar event rates for both groups. A recent multicenter randomized trial in China also showed that DCB was non-inferior to DES for 9-month insegment DS for small vessel disease [36].

Therefore, when compared with DES implantation, the overall non-inferior outcomes of $\mathrm{DCB}$ treatment support the use of DCB for the treatment of de novo small vessel disease (diameter of $2 \mathrm{~mm}$ to less than $3 \mathrm{~mm}$ ).

Large vessel disease. For large de novo coronary vessels, although the evidence is limited, recently published data demonstrated that fractional flow reserve (FFR) guided DCB treatment was safe and effective for de novo large coronary vessels including acute coronary syndrome with good anatomical and physiological patency at 9-month follow-up [37]. All cases used DCB of $2.5 \mathrm{~mm}$ or more in size and DCB of $3.0 \mathrm{~mm}$ or more in $70 \%$ cases. In this trial, if FFR after BA was favorable $(\geq 0.85)$, DCB was applied and if FFR after BA was $<0.85$, DES implantation was preferred. LLL with DCB was superior to DES $(0.05 \pm 0.27 \mathrm{~mm}$ in DCB vs. $0.40 \pm 0.54 \mathrm{~mm}$ in DES, $p=0.015$ ), and the FFR at 9-month follow-up did not differ between the two groups. In addition, using intravascular ultrasound (IVUS) and optical coherence tomography (OCT), the investigators suggested that DCB restores coronary blood flow by means of plaque modification, causing an increment in the minimal lumen area [38, 39]. Recent European data also showed that the FFR-guided DCB-only approach of de novo lesions (cutoff value of FFR: 0.80) was feasible and safe in stable CAD, showing positive remodeling without lumen loss by OCT at 6 months [40]. Another study showed that the safety and efficacy of DCB was comparable with DES when the cut-off value of FFR was 0.75 after balloon angioplasty [41]. Recent Chinese data showed that DCB for de novo coronary lesions with diameters greater than $2.8 \mathrm{~mm}$ was safe and effective as for small vessel lesions [42]. The follow-up MLD was significantly increased compared with immediate BA in both the large vessel group $(2.26 \pm 0.66 \mathrm{~mm}$ vs. $2.09 \pm 0.40 \mathrm{~mm}, \mathrm{p}=0.067)$ and the small vessel group $(1.75 \pm 0.48 \mathrm{~mm}$ vs. $1.58 \pm 0.31 \mathrm{~mm}, \mathrm{p}=0.008)$. These data suggested that DCB was also safe and effective in large de novo lesions. Nevertheless, a large multicenter tri$\mathrm{al}$ is needed to demonstrate the safety and efficacy of DCB treatment of the lesions of large vessels.
Others: Bifurcation, chronic total occlusion, diffuse long lesion, atherothrombotic lesion, calcified lesion etc. In bifurcation coronary diseases, current knowledge and experience suggest that treating lesions of the main vessel with a DES produces reasonable results but only suboptimal results in adjacent side-branches [43, 44]. Main vessel stenting in bifurcation lesion is associated with some disadvantages, such as overstretching of the distal vessel and straightening of the vessel, both leading to a carina shift into the side-branch $[2,45]$. Therefore, because DCB has an advantage of the absence of residual foreign material in the vessel, DCB treatment may be applied efficiently in bifurcation lesions. However, the randomized DEBIUT bifurcation trial comparing a DCB (Dior ${ }^{\mathrm{TM}}$ ) with BMS and DES failed to show angiographic and clinical superiority over BMS and DES using a provisional T-stenting technique [46]. Simply, DCB treatment was demonstrated to be safe with no thrombotic events, despite the shorter, 3-months duration of dual antiplatelet therapy (DAPT). The observational PEPCAD V study using DCB for both the main vessel and the side-branch resulted in a low LLL in the main vessel and the side-branch with a procedural success rate of $100 \%$ [47]. However, when the DCB was combined with BMS in the main vessel, late and very late stent thrombosis occurred in $3(7.5 \%)$ patients. The randomized BABILON trial included 108 patients with sequential main vessel/side-branch dilatation with DCB; provisional T-stenting with BMS in the main vessel was performed in the DCB group and performed with everolimus DES in the DES group [48]. Although the DCB plus provisional BMS strategy resulted in greater LLL and increased MACE compared to the DES group due to higher main vessel restenosis, both strategies showed similar results in the side-branch: LLL in the side-branch was $-0.04 \pm 0.76 \mathrm{~mm}$ in the case of BMS in the main vessel and $-0.03 \pm 0.51 \mathrm{~mm}$ in the case of DES in the main vessel $(\mathrm{p}=0.983)$. The recent randomized PEPCAD-BIF trial comparing the DCB-only approach with BA in the side-branch showed that the restenosis rate was $6 \%$ in the DCB group and $26 \%$ in the BA group $(\mathrm{p}=0.045)$ [49]. The Korean OCT study using the DCB-only approach applied only to the main vessel suggested that the DCB treatment was safe in bifurcation lesion and there was an increase in the side-branch ostium lumen enlargement despite the absence of treatment of the side-branch [45]. The mean side-branch ostial lumen area increased at 9-month follow-up. Although the optimal strategy and the role 
of DCB treatment in bifurcation diseases are not yet confirmed, DCB treatment may be an alternative option for bifurcation lesions. Approximately, these recommendations are similar to that of the German consensus [2]. The first-step is the pre-dilatation of the main branch and/or the side-branch using conventional balloons with a balloon-to-vessel ratio of 0.8-1.0 and an inflation pressure higher than nominal. If the flow-limiting dissection is absent and residual stenosis is $<30 \%$ in the main vessel and $<75 \%$ in the side-branch with thrombolysis in myocardial infarction (TIMI) flow $3, \mathrm{DCB}$ can be applied to the side-branch extending 4-5 $\mathrm{mm}$ into main vessel and distally $2-3 \mathrm{~mm}$ beyond the predilated area with a balloon-to-vessel ratio of $0.8-1.0$ at least for $60 \mathrm{~s}$. Then the DCB can be applied to the main vessel in the same way, extending the balloon covered length $2-3 \mathrm{~mm}$ on both sides, respectively beyond the pre-dilated area. If the result is not satisfactory, the DES can be applied to the main vessel and the provisional stenting can be applied to the side-branch. In other words, the application of a DES in the main vessel and a DCB-only approach in the side-branch may be reasonable and has been shown to be effective according to previous evidence, despite the need of further scientific evaluations. Practically, the DCB application on the side-branch is recommended before stenting the main vessel, rather than after stenting the main vessel, because the drug on the DCB may get lost when crossing the stent strut. If a final kissing balloon angioplasty needs to be performed, it is recommended to use conventional balloons.

Chronic total occlusion (CTO) poses significant technical challenges in percutaneous coronary intervention (PCI), and the results on long term efficacy and safety are still limited. In PEPCAD CTO, BMS was performed on patients followed by DCB to the stented segment as well as beyond the stent edges after successful CTO recanalization in a native coronary artery [50]. Angiographic results and clinical endpoints in the BMS plus DCB group were no different from those of matched patients treated with a paclitaxel-eluting stent. Another recent study showed that a DCB-only strategy without stenting was a feasible and well-tolerated treatment method for CTO if the predilatation result was good [51]. CTO lesions have negative remodeled distal vessels because they have not had flow for a long time. After BA, antegrade flow increases and vessels become larger, requiring several weeks to several months. Therefore, stent sizing immediately after BA is easy to underestimate and could cause late stent malapposition.
However, once treated with $\mathrm{DCB}$, it is possible that the vessels will return to their original size over time, which is one of the greatest advantages of DCB treatment in CTO lesions.

For diffuse long lesions, the implantation of long metal devices in coronary vessels may impair the restoration of vasomotion in the stented segment, associated with ISR, stent thrombosis, and neoatherosclerosis, and limit access for coronary artery bypass graft [52-54]. In a retrospective study, patients treated with DCB either alone or in combination with DES were compared with those obtained from a cohort of patients with similar characteristics treated with DES alone [55]. The outcome rates for $\mathrm{DCB} \pm \mathrm{DES}$ were comparable to those with DES alone at 2-year follow-up (MACE: $20.8 \%$ vs. $22.7 \%, \mathrm{p}=0.74$; TVR: $14.8 \%$ vs. $11.5 \%$, $\mathrm{p}=0.44$; TLR: $9.6 \%$ vs. $9.3 \%, \mathrm{p}=0.84)$. Thus, DCB treatment may be an alternative and useful approach in diffuse de novo long lesions, either alone or in combination with DES.

ST-segment elevation myocardial infarction is the most representative disease of atherothrombotic lesions. Although stent implantation has significantly reduced repeat revascularization for STEMI, even DES did not result in lower rates of recurrent MI or death, when compared with balloon angioplasty alone or BMS [56-61]. An important limitation of stent implantation in patients with STEMI is the persistent risk of stent thrombosis or ISR [62-64]. In the majority of STEMI patients, rapid restoration of coronary flow is the main purpose of treatment, and this can be achieved by a combined approach of pharmacologic (antiplatelet and antithrombotic agents) and interventional (thrombus aspiration, balloon dilatation) treatments before stenting. Therefore, DCB treatment may be an optional therapeutic strategy in STEMI if coronary flow is restored and no significant residual stenosis persists after thrombus aspiration and balloon dilatation. A previous study showed that the DCB-only approach is safe and feasible in the setting of STEMI comparing newer generation DES (Biomime ${ }^{\mathrm{TM}}$ Sirolimus-Eluting Coronary Stent) and showed good clinical and angiographic outcomes in a 6-month follow-up period [65]. Late lumen loss in the DES group was $0.10 \pm 0.19 \mathrm{~mm}$ and $-0.09 \pm$ $\pm 0.09 \mathrm{~mm}$ in the DCB group $(\mathrm{p}<0.05)$, and MACE were reported in $5.4 \%$ of patients in the DES group and none in the DCB group (risk ratio [RR] 5.13, 95\% CI: $0.25-103.42, \mathrm{p}=0.29$ ). Other recent registry data showed that $\mathrm{DCB}$-only strategy with provisional stenting is a safe and efficient in de novo coronary lesions in acute coronary syndrome (ACS) 
[66]. Therefore, although there remains a need for larger randomized data confirming this issue, the DCB approach may be considered in STEMI if good angiographic results are obtained after thrombus aspiration and balloon dilatation.

Heavily calcified lesions are associated with poorer clinical outcomes due to incomplete stent expansion and malapposition because of the difficulty of adequately deploying the stent into the lesion. Ito et al. [67] demonstrated that DCB treatment shows acceptable MACE and TLR rates at 6-month follow-up for calcified lesions. The rates for TLR and MACE at 2 years were comparable between the calcified group and the non-calcified group. These favorable results may be explained by the exclusion of patients with significant residual stenosis and dissection following the preparation of lesions with rotational atherectomy and a noncompliant balloon as well as the use of IVUS and OCT. Therefore, in calcified lesions with DCB treatment, it is thought that rotational atherectomy and non-compliant balloons used prior to $\mathrm{DCB}$ treatment reduces the calcific burden, thus enhancing penetration of the drug into the vessel wall.

\section{Optimal lesion preparation}

The successful PCI for treatment of CAD is not just the resolution of epicardial coronary artery stenosis but the acquisition of adequate coronary blood flow. Optimal lesion preparation is of the utmost importance to obtain proper flow in the subtended myocardium and this is performed by BA. A previous study demonstrated that after BA percent stenosis, intimal tear or dissection and pressure gradient of $20 \mathrm{mmHg}$ or more are risk factors for acute closure [68]. Other studies reported that coronary dissection with a TIMI flow grade of 3 or uncomplicated and non-flow-limiting dissections are associated with favorable outcomes and predict a low restenosis rate [69, 70]. Although $\mathrm{DCB}$ is similar in construction to conventional angioplasty, DCB is designed to deliver an anti-proliferative drug, not to relieve stenosis as BA does. Therefore, the key to successful DCB treatment depends on whether lesion preparation is appropriately performed before applying DCB. Optimal lesion preparation by pre-treating the lesion with conventional BA is considered the mandatory first step to obtain optimal results from DCB treatment. The main goals of pre-treatment are to improve blood flow by inducing dissection and to facilitate homogeneous drug delivery [71]. Although optimal lesion preparation is a very important factor for successful DCB treatment, there is a fear that major dissection may occur at a high rate. In this case, if flow-limiting dissection is developed, treatment ought to be performed with DES, and if flow is normal, it is safe to decide which device (DCB or DES) to select for treatment using FFR.

\section{In-stent restenosis (Fig. 1)}

Data recently published in Korea suggested that the independent predictors of target lesion failure (TLF) in patients with DES-ISR treated with DCB were residual DS after lesion preparation, DCB-to-stent size ratio, and DCB inflation time, whose best cutoff values were $20 \%, 0.91$, and $60 \mathrm{~s}$, respectively [72]. TLF rates were significantly higher in groups with residual DS $\geq 20 \%$ (34.7\% vs. $12.5 \%$; HR: 2.15 ; $95 \%$ CI: $1.86-2.48, \mathrm{p}<0.001)$, DCB-to-stent size ratio $\leq 0.91$ (46.4\% vs. $21.9 \%$; HR: 2.02 ; 95\% CI: $1.75-2.34, \mathrm{p}<0.001)$, and DCB inflation time $\leq 60 \mathrm{~s}(26.2 \%$ vs. $14.0 \%$; HR: 1.82 ; 95\% CI: $1.36-2.45, \mathrm{p}<0.001)$. When ISR lesions were classified by the combination of procedurerelated factors, TLF occurred in $8.3 \%$ in the fully optimized procedure group (residual DS $<20 \%$, DCB-to-stent size ratio $>0.91$, and inflation time $>60 \mathrm{~s}$ ) and $66.7 \%$ in the non-optimized group $(p<0.001)$. Unlike DES, the efficacy of DCB is proportional to the amount of the drug delivered to the target lesion. In this regard, the DCB delivery time (time delay from vascular access to the lesion) would correlate with the amount of drug loss into the bloodstream, and the lesion preparation status would affect distribution and absorption of the drug [73]. Therefore, fully optimized DCB treatment with optimal lesion preparation, fast delivery, and prolonged inflation time may play an important role in reducing TLF after DCB treatment.

The originally recommended form of lesion preparation is conventional angioplasty with a nonor semi-compliant balloon with a balloon-to-stent ratio of 0.9 and an inflation pressure higher than nominal [74]. Especially, the use of a non-compliant balloon is preferred over a semi-compliant balloon because a non-compliant balloon helps to improve the previous stent expansion. If the result from the use of a non- or semi-compliant balloon is not satisfactory, using a high-pressure non-compliant balloon or scoring/cutting the balloon may be recommended to facilitate a complete expansion of the restenosed previous stent, neointimal modification, and homogeneous drug delivery, which improves the antirestenotic efficacy of DCB therapy [26].

Based on scientific evidence, additional intravascular imaging such as IVUS and OCT is recommended 


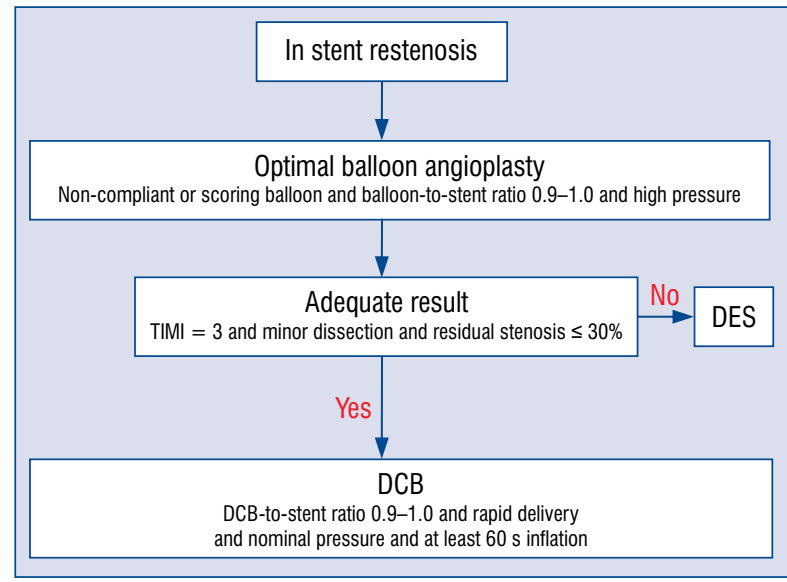

Figure 1. Treatment recommendations for in-stent restenosis. This flow chart shows the recommendations of drug-coated balloon (DCB) treatment for instent restenosis. If a non-compliant or scoring balloon or cutting balloon does not pass into the lesion, balloon angioplasty with a smaller sized semi-compliant balloon can be applied; DES - drug eluting stent.

to identify the morphological causes of the ISR and achieve optimal lesion preparation, satisfactory angiographic results and successful drug delivery.

\section{De novo lesion (Fig. 2)}

Lesion preparation is considered the mandatory first step for successful DCB treatment [74]. The simplest form of lesion preparation is conventional angioplasty with a semi-compliant balloon with a balloon-to-vessel ratio of $0.8-1.0$ and an inflation pressure higher than nominal. In more complex lesions, the use of non-compliant high-pressure balloons, scoring or cutting balloons, even rotablation might be considered as well as additional intravascular imaging (IVUS, OCT) or functional measurements (FFR) [2]. To determine whether it is appropriate to perform DCB treatment, all of the following three criteria have to be met after balloon angioplasty; no dissection or type A/B dissection, TIMI grade 3 flow, and residual stenosis $\leq 30 \%$. For larger vessels, when FFR was applied after BA it can determine whether treatment of this lesion with DCB or DES is appropriate. In the FFR-guided DCB approach, if the FFR value after BA is good enough to treat with $\mathrm{DCB}, \mathrm{DCB}$ treatment is safe and effective with good anatomical and physiological patency at follow-up [37]. In this study, if FFR after BA was $\geq 0.85$, DCB was applied and if FFR after BA was $<0.85$, stent implantation was preferred over DCB. Additionally, when the same criteria of lesion preparation using FFR were ap-

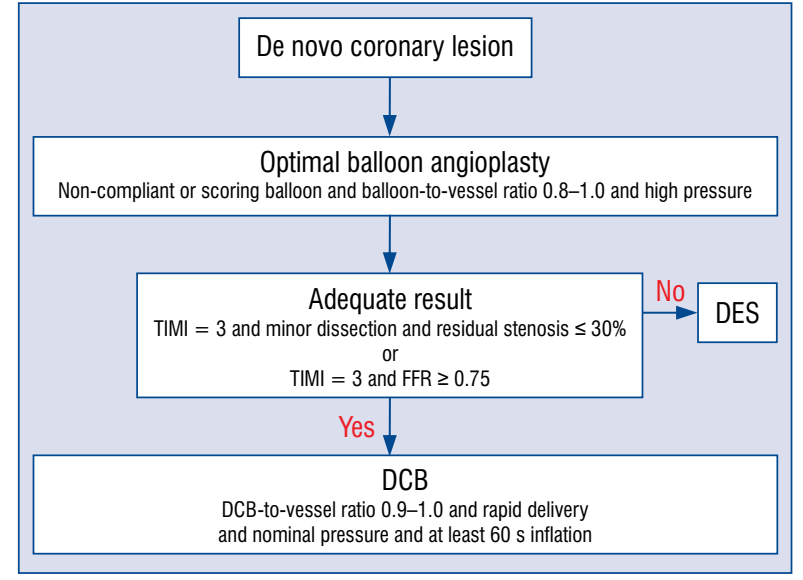

Figure 2. Treatment recommendations for de novo lesions. This flow chart shows the recommendations of drug-coated balloon (DCB) treatment for de novo lesion. If a non-compliant or scoring balloon or cutting balloon does not pass into the lesion, balloon angioplasty with a smaller sized semi-compliant balloon can be applied; DES - drug eluting stent.

plied, DCB treatment showed persistent anatomical and physiological patency with plaque redistribution and vessel remodeling without chronic elastic recoil or plaque compositional change during follow-up [38]. Similarly, recent European data showed that if it was determined that there was sufficient lesion preparation with residual stenosis $<40 \%$, FFR $>0.80$ and no severe dissection, DCB treatment was feasible and safe with a trend toward positive vessel remodeling without lumen loss at 6 months [40].

If the angiographic results are acceptable, $\mathrm{DCB}$ may be applied. It should extend beyond the predilated area by $2-3 \mathrm{~mm}$ on each side to avoid geographic mismatch. The diameter of the DCB should match with the diameter of the target vessel and the reference ratio of balloon to vessel is between 0.8 and 1.0. The DCB should be inflated for at least $60 \mathrm{~s}$ (30 s may be acceptable if the patient is intolerable to longer inflation time) at nominal pressure (about 7-8 atm) to avoid further dissection. It is important to remember that the DCB is a tool for drug delivery and not intended for resolving vessel stenosis like BA. Basically, DCB exists only for drug delivery and is not for angioplasty. Although a product company recommends that the $\mathrm{DCB}$ delivery time to the lesion should be within $2 \mathrm{~min}$, the faster, the better.

\section{Optimal medical treatment}

Previous trials showed a broad range of duration of DAPT, ranging from 1 to 12 months after 
DCB treatment. Although recent ESC guidelines recommend that DAPT should be considered for 6 months after DCB treatment in stable CAD [3], this recommendation did not reflect the results of recent trials for DCB treatment. Importantly, in the recent studies using relatively shorter duration of DAPT such as 1-3 months after DCB, there appeared to be no significant increase in MACE compared to cases with a longer duration of DAPT [29, 30, $32,46]$. In a large randomized BASKET-SMALL 2 trial comparing DCB with second-generation DES in small native $\mathrm{CAD}, \mathrm{DCB}$ was non-inferior to DES regarding 12-month composite clinical endpoint of MACE despite the shorter duration of 1-month DAPT after DCB treatment [33]. The advantages of shorter duration of DAPT after DCB treatment may be applicable to patients with high bleeding risk, an urgent surgical indication. According to the ESC guidelines for myocardial revascularization, all patients scheduled for DCB treatment should be considered for pre-treatment with acetylsalicylic acid (ASA) and clopidogrel [3]. To ensure full antiplatelet therapy, ASA with a loading dose of 150-300 mg and clopidogrel with a loading dose of $300 \mathrm{mg}$ should be initiated at least $6-16$ hours prior to the procedure [2]. If this is not possible, a loading dose of $600 \mathrm{mg}$ of clopidogrel should be applied at least 2 hours before the procedure. For ACS, the loading dose of the adenosine diphosphate (ADP)-receptor blocker (clopidogrel $600 \mathrm{mg}$ or prasugrel $60 \mathrm{mg}$ or ticagrelor $180 \mathrm{mg}$ ) should be administered as soon as possible. The duration of DAPT after DCB treatment varies depending on the indications of DCB. For treatment of BMS-ISR and DES-ISR, patients should maintain a lifelong therapy with ASA $100 \mathrm{mg}$ and take clopidogrel $75 \mathrm{mg}$ for at least $1-3$ months. For treatment of de novo coronary disease except ACS with DCB only, patients should receive ASA $100 \mathrm{mg}$ lifelong and clopidogrel $75 \mathrm{mg}$ for at least 1 month. In addition, for the cases of de novo stable coronary disease with DCB plus bail-out BMS, DAPT is recommended for at least 3-6 months. For patients with ACS, DAPT is recommended for at least 12 months regardless of the use of BMS or $\mathrm{DCB}$ or DES. For the treatment of bifurcation disease, if the DCB-only method without stenting is used, the duration of DAPT should be the same as in the case of other de novo coronary diseases. For cases using DCB plus stenting, the recommended duration of DAPT is at least $6-12$ months because of the higher risk of stent thrombosis.

Furthermore, the effect of high-dose statin therapy can be extended after DCB application. In regards to the effects of statin therapy, a previous study demon- strated that a clear reduction of lipid core was observed only for the thin-cap fibroatheroma plaque type, suggesting that changes in plaque composition following statin therapy might occur earlier in vulnerable plaque than in stable plaque [75]. Recent Korean data showed that DCB treatment with high dose statin caused persistent patency with plaque redistribution without chronic elastic recoil and restored coronary blood flow resulting in increased lumen areas at follow-up [38]. In other words, it can be expected that there will be regression of plaque after DCB treatment through high dose statin therapy because balloon angioplasty replaces stable plaque with iatrogenic vulnerable plaque. Therefore, the high intensity of statin therapy can reinforce the efficacy of DCB treatment.

\section{Perspectives}

Most recent researches have focused on different coatings and drug delivery technologies. Although zotarolimus- and sirolimus-DCB have shown promising results in preclinical studies, it remains to be determined whether they will result in relevant clinical effects [76-78]. In comparison to zotarolimus-eluting stents, zotarolimus-coated balloons demonstrated similar reductions in neointimal proliferation with a reduction in inflammation scores [77]. A trial of sirolimus-DCB showed similar angiographic outcomes in the treatment of DES-ISR compared with a clinically proven paclitaxel-DCB [79]. Another study of a novel phospholipid-encapsulated sirolimus nanocarrier, used as a coating, showed the most appropriate identification of the best nanoparticle structure associated with an extremely efficient transfer of the drug to all layers of the vessel wall, achieving high tissue concentrations that persisted days after the application with low systemic drug leaks [78]. Furthermore, a recent preclinical study suggested that the vascular effects of sirolimus nanoparticles delivered through a porous angioplasty balloon in a porcine model achieved therapeutic long-term intra-arterial drug concentrations without significant systemic residual exposure [80]. These different types of DCB are unique (i.e., drug type, drug dose, crystallinity, and excipient) with different clinical outcomes. Further research with head-to-head comparison between different $\mathrm{DCBs}$ in patients with $\mathrm{CAD}$ is needed to determine which DCBs are most effective.

\section{Conclusions}

Drug-coated balloons treatment is an attractive therapeutic option and may have several ben- 
efits over stent-based strategies in various subsets of coronary diseases. DCB treatment is vastly superior to BA for both ISR and de novo coronary disease. At present, DCB and second-generation DES are recommended in both BMS-ISR and DES-ISR. Although DES has shown the best angiographic and clinical outcomes, DCB may be an alternative option for ISR lesion due to favorable outcomes similar to those of DES, without adding a new stent layer. For de novo coronary disease, although DCB treatment remains controversial in various settings, a provisional $\mathrm{DCB}$ approach after optimal BA may have advantages over a direct DCB approach. For successful DCB treatment, the first and most important step is optimal lesion preparation using conventional $\mathrm{BA}$, preferably with the use of a non-compliant balloon or scoring balloon. If the result is no flow-limiting dissection and acceptable residual DS and normal flow, a DCB-only approach without any vascular scaffold like a stent is recommended. In addition, for more complex lesions such as bifurcation disease and larger de novo coronary lesions, further intravascular imaging or functional measurements are more useful than a simple angiography-guided strategy. There is insufficient data about the ideal duration of DAPT in DCB treatment but it is certain that a relatively shorter duration of DAPT than that required in DES would be reasonable. In terms of prognosis, high-dose statin therapy is expected to improve the efficacy of DCB treatment. Finally, if its applications are carefully and adequately performed with a good technique, DCB may have an important role in the treatment of ISR and in various de novo coronary lesions.

\section{Conflict of interest: None declared}

\section{References}

1. Scheller B, Speck U, Abramjuk C, et al. Paclitaxel balloon coating, a novel method for prevention and therapy of restenosis. Circulation. 2004; 110(7): 810-814, doi: 10.1161/01. CIR.0000138929.71660.E0, indexed in Pubmed: 15302790.

2. Kleber FX, Rittger H, Bonaventura K, et al. Drug-coated balloons for treatment of coronary artery disease: updated recommendations from a consensus group. Clin Res Cardiol. 2013; 102(11): 785-797, doi: 10.1007/s00392-013-0609-7, indexed in Pubmed: 23982467.

3. Neumann FJ, Sousa-Uva M, Ahlsson A, et al. 2018 ESC/EACTS Guidelines on myocardial revascularization. EuroIntervention. 2019; 14(14): 1435-1534, doi: 10.4244/eijy19m01_01.

4. Ong PJ, Zeymer U, Waliszewski M, et al. Differences in clinical and angiographic profiles between Asian and Western patients with coronary artery disease: insights from the prospective „real world" paclitaxel-coated balloon registry. Int J Cardiol. 2014;
175(1): 199-200, doi: 10.1016/j.ijcard.2014.04.239, indexed in Pubmed: 24820752.

5. Akiyama T, Moussa I, Reimers B, et al. Angiographic and clinical outcome following coronary stenting of small vessels: a comparison with coronary stenting of large vessels. J Am Coll Cardiol. 1998; 32(6): 1610-1618, doi: 10.1016/s0735-1097(98)00444-6, indexed in Pubmed: 9822086.

6. Hori M, Connolly SJ, Ezekowitz MD, et al. Efficacy and safety of dabigatran vs. warfarin in patients with atrial fibrillation: subanalysis in Japanese population in RE-LY trial. Circ J. 2011; 75(4): 800-805, doi: 10.1253/circj.cj-11-0191, indexed in Pubmed: 21436594 .

7. Kohsaka S, Kimura T, Goto M, et al. Difference in patient profiles and outcomes in Japanese versus American patients undergoing coronary revascularization (collaborative study by CREDO-Kyoto and the Texas Heart Institute Research Database). Am J Cardiol. 2010; 105(12): 1698-1704, doi: 10.1016/j. amjcard.2010.01.349, indexed in Pubmed: 20538117.

8. Misumida N, Ogunbayo GO, Kim SM, et al. Higher risk of bleeding in asians presenting with st-segment elevation myocardial infarction: analysis of the national inpatient sample database. Angiology. 2018; 69(6): 548-554, doi: 10.1177/0003319717730168, indexed in Pubmed: 28905638.

9. Wöhrle J, Zadura M, Möbius-Winkler S, et al. SeQuentPlease World Wide Registry: clinical results of SeQuent please paclitaxel-coated balloon angioplasty in a large-scale, prospective registry study. J Am Coll Cardiol. 2012; 60(18): 1733-1738, doi: 10.1016/j.jacc.2012.07.040, indexed in Pubmed: 23040575.

10. Mehilli J, Byrne RA, Tiroch K, et al. Randomized trial of paclitaxel- versus sirolimus-eluting stents for treatment of coronary restenosis in sirolimus-eluting stents: the ISAR-DESIRE 2 (Intracoronary Stenting and Angiographic Results: Drug Eluting Stents for In-Stent Restenosis 2) study. J Am Coll Cardiol. 2010; 55(24): 2710-2716, doi: 10.1016/j.jacc.2010.02.009, indexed in Pubmed: 20226618.

11. Abizaid A, Costa JR, Banning A, et al. The sirolimus-eluting Cypher Select coronary stent for the treatment of bare-metal and drug-eluting stent restenosis: insights from the e-SELECT (Multicenter Post-Market Surveillance) registry. JACC Cardiovasc Interv. 2012; 5(1): 64-71, doi: 10.1016/j.jcin.2011.09.016, indexed in Pubmed: 22230152.

12. Alli OO, Teirstein PS, Satler L, et al. Five-year follow-up of the Sirolimus-Eluting Stents vs Vascular Brachytherapy for Bare Metal In-Stent Restenosis (SISR) trial. Am Heart J. 2012; 163(3): 438-445, doi: 10.1016/j.ahj.2011.11.019, indexed in Pubmed: 22424015.

13. Scheller B, Hehrlein C, Bocksch W, et al. Treatment of Coronary In-Stent Restenosis with a Paclitaxel-Coated Balloon Catheter. N Engl J Med. 2006; 355(20): 2113-2124, doi: 10.1056/ nejmoa061254.

14. Unverdorben M, Vallbracht C, Cremers B, et al. Paclitaxel-coated balloon catheter versus paclitaxel-coated stent for the treatment of coronary in-stent restenosis. Circulation. 2009; 119(23): 29862994, doi: 10.1161/CIRCULATIONAHA.108.839282, indexed in Pubmed: 19487593.

15. Alfonso F, Pérez-Vizcayno MJ, Cárdenas A, et al. A randomized comparison of drug-eluting balloon versus everolimus-eluting stent in patients with bare-metal stent-in-stent restenosis: the RIBS V Clinical Trial (Restenosis Intra-stent of Bare Metal Stents: paclitaxel-eluting balloon vs. everolimus-eluting stent). J Am Coll Cardiol. 2014; 63(14): 1378-1386, doi: 10.1016/j. jacc.2013.12.006, indexed in Pubmed: 24412457. 
16. Habara S, Mitsudo K, Kadota K, et al. Effectiveness of paclitaxel-eluting balloon catheter in patients with sirolimus-eluting stent restenosis. JACC Cardiovasc Interv. 2011; 4(2): 149-154, doi: 10.1016/j.jcin.2010.10.012, indexed in Pubmed: 21349452.

17. Rittger H, Brachmann J, Sinha AM, et al. A randomized, multicenter, single-blinded trial comparing paclitaxel-coated balloon angioplasty with plain balloon angioplasty in drug-eluting stent restenosis: the PEPCAD-DES study. J Am Coll Cardiol. 2012; 59(15): 1377-1382, doi: 10.1016/j.jacc.2012.01.015, indexed in Pubmed: 22386286.

18. Xu Bo, Gao R, Wang J, et al. A prospective, multicenter, randomized trial of paclitaxel-coated balloon versus paclitaxel-eluting stent for the treatment of drug-eluting stent in-stent restenosis: results from the PEPCAD China ISR trial. JACC Cardiovasc Interv. 2014; 7(2): 204-211, doi: 10.1016/j.jcin.2013.08.011, indexed in Pubmed: 24556098.

19. Byrne RA, Neumann FJ, Mehilli J, et al. Paclitaxel-eluting balloons, paclitaxel-eluting stents, and balloon angioplasty in patients with restenosis after implantation of a drug-eluting stent (ISAR-DESIRE 3): a randomised, open-label trial. Lancet. 2013; 381(9865): 461-467, doi: 10.1016/S0140-6736(12)61964-3, indexed in Pubmed: 23206837.

20. Indermuehle A, Bahl R, Lansky AJ, et al. Drug-eluting balloon angioplasty for in-stent restenosis: a systematic review and meta-analysis of randomised controlled trials. Heart. 2013; 99(5): 327-333, doi: 10.1136/heartjnl-2012-302945, indexed in Pubmed: 23335497.

21. Liu L, Liu B, Ren J, et al. Comparison of drug-eluting balloon versus drug-eluting stent for treatment of coronary artery disease: a meta-analysis of randomized controlled trials. BMC Cardiovasc Disord. 2018; 18(1): 46, doi: 10.1186/s12872-018-0771-y, indexed in Pubmed: 29499651.

22. Lee JM, Park J, Kang J, et al. Comparison among drug-eluting balloon, drug-eluting stent, and plain balloon angioplasty for the treatment of in-stent restenosis: a network meta-analysis of 11 randomized, controlled trials. JACC Cardiovasc Interv. 2015; 8(3): 382-394, doi: 10.1016/j.jcin.2014.09.023, indexed in Pubmed: 25703886.

23. Alfonso F, Pérez-Vizcayno MJ, Cárdenas A, et al. A prospective randomized trial of drug-eluting balloons versus everolimuseluting stents in patients with in-stent restenosis of drug-eluting stents: the RIBS IV randomized clinical trial. J Am Coll Cardiol. 2015; 66(1): 23-33, doi: 10.1016/j.jacc.2015.04.063, indexed in Pubmed: 26139054.

24. Baan J, Claessen BE, Dijk KBv, et al. A randomized comparison of paclitaxel-eluting balloon versus everolimus-eluting stent for the treatment of any in-stent restenosis: the DARE trial. JACC Cardiovasc Interv. 2018; 11(3): 275-283, doi: 10.1016/j. jcin.2017.10.024, indexed in Pubmed: 29413242.

25. Kawamoto H, Ruparelia N, Latib A, et al. Drug-Coated balloons versus second-generation drug-eluting stents for the management of recurrent multimetal-layered in-stent restenosis. JACC Cardiovasc Interv. 2015; 8(12): 1586-1594, doi: 10.1016/j. jcin.2015.04.032, indexed in Pubmed: 26386758.

26. Kufner S, Joner M, Schneider S, et al. Neointimal modification with scoring balloon and efficacy of drug-coated balloon therapy in patients with restenosis in drug-eluting coronary stents: a randomized controlled trial. JACC Cardiovasc Interv. 2017; 10(13): 1332-1340, doi: 10.1016/j.jcin.2017.04.024, indexed in Pubmed: 28683939.

27. Belkacemi A, Agostoni P, Nathoe HM, et al. First results of the DEB-AMI (drug eluting balloon in acute ST-segment elevation myocardial infarction) trial: a multicenter randomized comparison of drug-eluting balloon plus bare-metal stent versus baremetal stent versus drug-eluting stent in primary percutaneous coronary intervention with 6-month angiographic, intravascular, functional, and clinical outcomes. J Am Coll Cardiol. 2012; 59(25): 2327-2337, doi: 10.1016/j.jacc.2012.02.027, indexed in Pubmed: 22503057.

28. Fischer D, Scheller B, Schäfer A, et al. Paclitaxcel-coated balloon plus bare metal stent vs. sirolimus-eluting stent in de novo lesions: an IVUS study. EuroIntervention. 2012; 8(4): 450-455, doi: 10.4244/EIJV8I4A71, indexed in Pubmed: 22917728.

29. Cortese B, Micheli A, Picchi A, et al. Paclitaxel-coated balloon versus drug-eluting stent during $\mathrm{PCI}$ of small coronary vessels, a prospective randomised clinical trial. The PICCOLETO study. Heart. 2010; 96(16): 1291-1296, doi: 10.1136/hrt.2010.195057, indexed in Pubmed: 20659948.

30. Latib A, Colombo A, Castriota F, et al. A randomized multicenter study comparing a paclitaxel drug-eluting balloon with a paclitaxel-eluting stent in small coronary vessels. J Am Coll Cardiol. 2012; 60(24): 2473-2480, doi: 10.1016/j.jacc.2012.09.020.

31. Zeymer U, Waliszewski M, Spiecker M, et al. Prospective, real world' registry for the use of the ,PCB only' strategy in small vessel de novo lesions. Heart. 2014; 100(4): 311-316, doi: 10.1136/ heartjnl-2013-304881, indexed in Pubmed: 24281754.

32. Unverdorben M, Kleber FX, Heuer H, et al. Treatment of small coronary arteries with a paclitaxel-coated balloon catheter in the PEPCAD I study: are lesions clinically stable from 12 to 36 months? EuroIntervention. 2013; 9(5): 620-628, doi: 10.4244/ EIJV9I5A99, indexed in Pubmed: 24058078.

33. Jeger RV, Farah A, Ohlow MA, et al. Drug-coated balloons for small coronary artery disease (BASKET-SMALL 2): an openlabel randomised non-inferiority trial. Lancet. 2018; 392(10150): 849-856, doi: 10.1016/S0140-6736(18)31719-7, indexed in Pubmed: 30170854.

34. Mitomo S, Jabbour RJ, Mangieri A, et al. Mid-term clinical outcomes after bailout drug-eluting stenting for suboptimal drugcoated balloon results: Insights from a Milan registry. Int J Cardiol. 2018; 263: 17-23, doi: 10.1016/j.ijcard.2018.04.050, indexed in Pubmed: 29685691.

35. Mok KH, Wickramarachchi U, Watson T, et al. Safety of bailout stenting after paclitaxel-coated balloon angioplasty. Herz. 2017; 42(7): 684-689, doi: 10.1007/s00059-016-4502-9, indexed in Pubmed: 27858114 .

36. Tang Y, Qiao S, Su Xi, et al. Drug-Coated balloon versus drugeluting stent for small-vessel disease: the RESTORE SVD china randomized trial. JACC Cardiovasc Interv. 2018; 11(23): 2381-2392, doi: 10.1016/j.jcin.2018.09.009, indexed in Pubmed: 30522667.

37. Shin ES, Ann SH, Balbir Singh G, et al. Fractional flow reserveguided paclitaxel-coated balloon treatment for de novo coronary lesions. Catheter Cardiovasc Interv. 2016; 88(2): 193-200, doi: 10.1002/ccd.26257, indexed in Pubmed: 26423017.

38. Ann SH, Balbir Singh G, Lim KH, et al. Anatomical and physiological changes after paclitaxel-coated balloon for atherosclerotic de novo coronary lesions: serial IVUS-VH and FFR study. PLoS One. 2016; 11(1): e0147057, doi: 10.1371/journal.pone.0147057, indexed in Pubmed: 26824602.

39. Ann SH, Her AY, Singh GB, et al. Serial morphological and functional assessment of the paclitaxel-coated balloon for de novo lesions. Rev Esp Cardiol (Engl Ed). 2016; 69(11): 1026-1032, doi: 10.1016/j.rec.2016.03.026, indexed in Pubmed: 27321644. 
40. Poerner TC, Duderstadt C, Goebel B, et al. Fractional flow reserve-guided coronary angioplasty using paclitaxel-coated balloons without stent implantation: feasibility, safety and 6-month results by angiography and optical coherence tomography. Clin Res Cardiol. 2017; 106(1): 18-27, doi: 10.1007/s00392-016-1019-4, indexed in Pubmed: 27379610.

41. Her AY, Shin ES, Lee JM, et al. Paclitaxel-coated balloon treatment for functionally nonsignificant residual coronary lesions after balloon angioplasty. Int J Cardiovasc Imaging. 2018; 34(9): 1339-1347, doi: 10.1007/s10554-018-1351-z, indexed in Pubmed: 29696453.

42. Yu X, Ji F, Xu F, et al. Treatment of large de novo coronary lesions with paclitaxel-coated balloon only: results from a Chinese institute. Clin Res Cardiol. 2019; 108(3): 234-243, doi: 10.1007/ s00392-018-1346-8, indexed in Pubmed: 30074078.

43. Steigen TK, Maeng M, Wiseth R, et al. Randomized study on simple versus complex stenting of coronary artery bifurcation lesions: the Nordic bifurcation study. Circulation. 2006; 114(18): 1955-1961, doi: 10.1161/CIRCULATIONAHA.106.664920, indexed in Pubmed: 17060387.

44. Koo BK, Kang HJ, Youn TJ, et al. Physiologic assessment of jailed side branch lesions using fractional flow reserve. J Am Coll Cardiol. 2005; 46(4): 633-637, doi: 10.1016/j.jacc.2005.04.054, indexed in Pubmed: 16098427.

45. Her AY, Ann SH, Singh GB, et al. Serial morphological changes of side-branch ostium after paclitaxel-coated balloon treatment of de novo coronary lesions of main vessels. Yonsei Med J. 2016; 57(3): 606-613, doi: 10.3349/ymj.2016.57.3.606, indexed in Pubmed: 26996558.

46. Stella PR, Belkacemi A, Dubois C, et al. A multicenter randomized comparison of drug-eluting balloon plus bare-metal stent versus bare-metal stent versus drug-eluting stent in bifurcation lesions treated with a single-stenting technique: six-month angiographic and 12-month clinical results of the drug-eluting balloon in bifurcations trial. Catheter Cardiovasc Interv. 2012; 80(7): 1138-1146, doi: 10.1002/ccd.23499, indexed in Pubmed: 22422607.

47. Mathey DG, Wendig I, Boxberger M, et al. Treatment of bifurcation lesions with a drug-eluting balloon: the PEPCAD V (Paclitaxel Eluting PTCA Balloon in Coronary Artery Disease) trial. EuroIntervention. 2011; 7 Suppl K: K61-K65, doi: 10.4244/ EIJV7SKA11, indexed in Pubmed: 22027730.

48. Lopez Minguez JR, Nogales Asensio JM, Doncel Vecino LJ, et al. A prospective randomised study of the paclitaxel-coated balloon catheter in bifurcated coronary lesions (BABILON trial): 24-month clinical and angiographic results. EuroIntervention. 2014; 10(1): 50-57, doi: 10.4244/EIJV10I1A10, indexed in Pubmed: 24832638 .

49. Kleber FX, Rittger H, Ludwig J, et al. Drug eluting balloons as stand alone procedure for coronary bifurcational lesions: results of the randomized multicenter PEPCAD-BIF trial. Clin Res Cardiol. 2016; 105(7): 613-621, doi: 10.1007/s00392-015-0957-6, indexed in Pubmed: 26768146.

50. Wöhrle J, Werner GS. Paclitaxel-coated balloon with bare-metal stenting in patients with chronic total occlusions in native coronary arteries. Catheter Cardiovasc Interv. 2013; 81(5): 793-799, doi: 10.1002/ccd.24409, indexed in Pubmed: 22511572.

51. Köln PJ, Scheller B, Liew HB, et al. Treatment of chronic total occlusions in native coronary arteries by drug-coated balloons without stenting - A feasibility and safety study. Int J Cardiol. 2016; 225: 262-267, doi: 10.1016/j.ijcard.2016.09.105, indexed in Pubmed: 27741486.
52. Hofma SH, van der Giessen WJ, van Dalen BM, et al. Indication of long-term endothelial dysfunction after sirolimus-eluting stent implantation. Eur Heart J. 2006; 27(2): 166-170, doi: 10.1093/ eurheartj/ehi571, indexed in Pubmed: 16249221.

53. Lee CH, Lee JY, Park GM, et al. Predictors of restenosis after placement of drug-eluting stents in one or more coronary arteries. Am J Cardiol. 2006; 97(4): 506-511, doi: 10.1016/j.amjcard.2005.09.084, indexed in Pubmed: 16461047.

54. D'Ascenzo F, Bollati M, Clementi F, et al. Incidence and predictors of coronary stent thrombosis: evidence from an international collaborative meta-analysis including 30 studies, 221,066 patients, and 4276 thromboses. Int J Cardiol. 2013; 167(2): 575-584, doi: 10.1016/j.ijcard.2012.01.080, indexed in Pubmed: 22360945.

55. Costopoulos C, Latib A, Naganuma T, et al. The role of drugeluting balloons alone or in combination with drug-eluting stents in the treatment of de novo diffuse coronary disease. JACC Cardiovasc Interv. 2013; 6(11): 1153-1159, doi: 10.1016/j. jcin.2013.07.005, indexed in Pubmed: 24262615.

56. Zhu MM, Feit A, Chadow H, et al. Primary stent implantation compared with primary balloon angioplasty for acute myocardial infarction: a meta-analysis of randomized clinical trials. Am J Cardiol. 2001; 88(3): 297-301, doi: 10.1016/s0002-9149(01)01645-9, indexed in Pubmed: 11472712.

57. De Luca G, Suryapranata H, Stone GW, et al. Coronary stenting versus balloon angioplasty for acute myocardial infarction: a meta-regression analysis of randomized trials. Int J Cardiol. 2008; 126(1): 37-44, doi: 10.1016/j.ijcard.2007.03.112, indexed in Pubmed: 17544528.

58. Suryapranata H, De Luca G, van ,t Hof AWJ, et al. Is routine stenting for acute myocardial infarction superior to balloon angioplasty? A randomised comparison in a large cohort of unselected patients. Heart. 2005; 91(5): 641-645, doi: 10.1136/ hrt.2004.056705, indexed in Pubmed: 15831652.

59. Laarman GJ, Suttorp MJ, Dirksen MT, et al. Paclitaxel-eluting versus uncoated stents in primary percutaneous coronary intervention. N Engl J Med. 2006; 355(11): 1105-1113, doi: 10.1056/ NEJMoa062598, indexed in Pubmed: 16971717.

60. Kastrati A, Dibra A, Spaulding C, et al. Meta-analysis of randomized trials on drug-eluting stents vs. bare-metal stents in patients with acute myocardial infarction. Eur Heart J. 2007; 28(22): 2706-2713, doi: 10.1093/eurheartj/ehm402, indexed in Pubmed: 17901079.

61. De Luca G, Smits P, Hofma SH, et al. Efficacy and safety of drug-eluting stents in ST-segment elevation myocardial infarction: a meta-analysis of randomized trials. Int J Cardiol. 2009; 133(2): 213-222, doi: $10.1016 /$ j.jijcard.2007.12.040, indexed in Pubmed: 18394731.

62. Nakazawa G, Finn AV, Joner M, et al. Delayed arterial healing and increased late stent thrombosis at culprit sites after drug-eluting stent placement for acute myocardial infarction patients: an autopsy study. Circulation. 2008; 118(11): 1138-1145, doi: 10.1161/CIRCULATIONAHA.107.762047, indexed in Pubmed: 18725485.

63. Stone SG, Serrao GW, Mehran R, et al. Incidence, predictors, and implications of reinfarction after primary percutaneous coronary intervention in ST-segment-elevation myocardial infarction: the Harmonizing Outcomes with Revascularization and Stents in Acute Myocardial Infarction Trial. Circ Cardiovasc Interv. 2014; 7(4): 543-551, doi: 10.1161/CIRCINTERVENTIONS.114.001360, indexed in Pubmed: 24939928.

64. Gonzalo N, Barlis P, Serruys PW, et al. Incomplete stent apposition and delayed tissue coverage are more frequent in drug- 
eluting stents implanted during primary percutaneous coronary intervention for ST-segment elevation myocardial infarction than in drug-eluting stents implanted for stable/unstable angina: insights from optical coherence tomography. JACC Cardiovasc Interv. 2009; 2(5): 445-452, doi: 10.1016/j.jcin.2009.01.012, indexed in Pubmed: 19463469.

65. Gobić D, Tomulić V, Lulić D, et al. Drug-Coated balloon versus drug-eluting stent in primary percutaneous coronary intervention: a feasibility study. Am J Med Sci. 2017; 354(6): 553-560, doi: 10.1016/j.amjms.2017.07.005, indexed in Pubmed: 29208251.

66. Uskela S, Kärkkäinen JM, Eränen J, et al. Percutaneous coronary intervention with drug-coated balloon-only strategy in stable coronary artery disease and in acute coronary syndromes: An allcomers registry study. Catheter Cardiovasc Interv. 2019; 93(5): 893-900, doi: 10.1002/ccd.27950, indexed in Pubmed: 30380186.

67. Ito R, Ueno $\mathrm{K}$, Yoshida $\mathrm{T}$, et al. Outcomes after drug-coated balloon treatment for patients with calcified coronary lesions. J Interv Cardiol. 2018; 31(4): 436-441, doi: 10.1111/joic.12484, indexed in Pubmed: 29266411.

68. Ellis SG, Roubin GS, King SB, et al. Angiographic and clinical predictors of acute closure after native vessel coronary angioplasty. Circulation. 1988; 77(2): 372-379, doi: 10.1161/01. cir.77.2.372, indexed in Pubmed: 2962787.

69. Cappelletti A, Margonato A, Rosano G, et al. Short- and longterm evolution of unstented nonocclusive coronary dissection after coronary angioplasty. J Am Coll Cardiol. 1999; 34(5): 1484-1488, doi: 10.1016/s0735-1097(99)00395-2, indexed in Pubmed: 10551696.

70. Leimgruber PP, Roubin GS, Anderson HV, et al. Influence of intimal dissection on restenosis after successful coronary angioplasty. Circulation. 1985; 72(3): 530-535, doi: 10.1161/01. cir.72.3.530, indexed in Pubmed: 3160507.

71. Huber MS, Mooney JF, Madison J, et al. Use of a morphologic classification to predict clinical outcome after dissection from coronary angioplasty. Am J Cardiol. 1991; 68(5): 467-471, doi: 10.1016/0002-9149(91)90780-o, indexed in Pubmed: 1872273.

72. Rhee TM, Lee JM, Shin ES, et al. Impact of optimized procedure-related factors in drug-eluting balloon angioplasty for treatment of in-stent restenosis. JACC Cardiovasc Interv. 2018;
11(10): 969-978, doi: 10.1016/j.jcin.2018.02.002, indexed in Pubmed: 29798774.

73. Kim HS, Rhee TM. Farewell to drug-eluting balloons for instent restonsis?: appropriate technique of drug-eluting balloons implantation matters. JACC Cardiovasc Interv. 2018; 11(10): 992-994, doi: 10.1016/j.jcin.2018.04.002, indexed in Pubmed: 29798777.

74. Kleber FX, Mathey DG, Rittger H, et al. How to use the drugeluting balloon: recommendations by the German consensus group. EuroIntervention. 2011; 7 Suppl K: K125-K128, doi: 10.4244/EIJV7SKA21, indexed in Pubmed: 22027722.

75. Hwang DS, Shin ES, Kim SJ, et al. Early differential changes in coronary plaque composition according to plaque stability following statin initiation in acute coronary syndrome: classification and analysis by intravascular ultrasound-virtual histology. Yonsei Med J. 2013; 54(2): 336-344, doi: 10.3349/ymj.2013.54.2.336, indexed in Pubmed: 23364965.

76. Clever YP, Peters D, Calisse J, et al. Novel sirolimus-coated balloon catheter: in vivo evaluation in a porcine coronary model. Circ Cardiovasc Interv. 2016; 9(4): e003543, doi: 10.1161/CIRCINTERVENTIONS.115.003543, indexed in Pubmed: 27069105.

77. Cremers B, Toner JL, Schwartz LB, et al. Inhibition of neointimal hyperplasia with a novel zotarolimus coated balloon catheter. Clin Res Cardiol. 2012; 101(6): 469-476, doi: 10.1007/s00392012-0415-7, indexed in Pubmed: 22293991.

78. Lemos PA, Farooq V, Takimura CK, et al. Emerging technologies: polymer-free phospholipid encapsulated sirolimus nanocarriers for the controlled release of drug from a stent-plus-balloon or a stand-alone balloon catheter. EuroIntervention. 2013; 9(1): 148-156, doi: 10.4244/EIJV9I1A21, indexed in Pubmed: 23685303.

79. Ali RM, Abdul Kader MA, Wan Ahmad WA, et al. Treatment of coronary drug-eluting stent restenosis by a sirolimus- or paclitaxel-coated balloon. JACC Cardiovasc Interv. 2019; 12(6): 558-566, doi: 10.1016/j.jcin.2018.11.040, indexed in Pubmed: 30898253.

80. Granada JF, Tellez A, Baumbach WR, et al. In vivo delivery and long-term tissue retention of nano-encapsulated sirolimus using a novel porous balloon angioplasty system. EuroIntervention. 2016; 12(6): 740-747, doi: 10.4244/EIJY15M10_01, indexed in Pubmed: 26428893. 